\title{
Medidor ultrasónico de nivel de agua para estanques
}

\author{
J. Gutiérrez y M.A. Porta-Gándara \\ Centro de Investigaciones Biológicas del N oroeste, La Paz, BCS \\ E-mails: joaquing04@ cibnor.mx y maporta@cibnor.mx
}

(Recibido: octubre de 2005; aceptado: mayo de 2006)

\begin{abstract}
Resumen
En este trabajo se reporta el diseño y desempeño de un medidor de nivel de agua ultrasónico de $40 \mathrm{kHz}$, contenido en una unidad remota para regis trar el tirante del agua en estanques acuícolas, con una resolución de $\pm 0.003 \mathrm{~m}$ y una variación del nivel de hasta $10 \mathrm{~m}$. La velocidad del sonido está compensada con la medición de la temperatura del medio de propagación mediante un sensor digital que utiliza el protocolo de comunicación 1-Wire ®. La información se presenta en una pantalla de cristal líquido y es trasmitida a una unidad local mediante el protocolo inalámbrico Bluetooth ${ }^{\circledR}$ para su monitoreo en tiempo real, mediante una interfaz gráfica del usuario programada en $M$ atlab ${ }^{\circledR}$ a través de un puerto serial universal (USB). A manera de respaldo, las mediciones se graban en una memoria de estado sólido EEPROM mediante el protocolo I2C ®. Este sistema se desarrolló con base en un microcontrolador comercial de bajo costo STAM P BS2p, diseñado para una amplia gama de aplicaciones, el cual utiliza un lenguaje propio de programación de alto nivel para manejar directamente los puertos de entrada/salida $(/ / 0)$. El equipo satisface las necesidades de medición automática del nivel de agua en estanques acuícolas para la supervisión y control.
\end{abstract}

Descriptores: Medición de nivel, estanques acuícolas, ultrasónico, evaporación.

\begin{abstract}
In this work thedesign, con struction and per for mance of $40 \mathrm{kHz}$ ul tra sonicwa ter level measurer is reported. Em bedded into a remote unit, it is used to mea surethewa ter level in aquaculture ponds. A res 0 lu tion of $\pm 0,003 \mathrm{~m}$ is at tained with a mea sure ment range up to $10 \mathrm{~m}$. The sound speed is com pensated with themea sure ments of theam bi ent tem per a tureby means of a dig $i$ tal sen sor that uses the 1-Wire $\circledast$ com mu ni ca tion pro to col. Thein for ma tion is dis played in a $L C D$ and sent to a lo cal unitby means of the B luetooth $®$ wireless pro to col. A graph $i$ cal user in ter facepro grammed in $M$ atlab $®$ through a USB port al lows real timemon i tor ing. An EE PRO M is con nected by thel2C ®protocol and used as data backup. This sys tem was devel oped with themicrocontroller STAM P BS $2 p$ designed for an am plerange of ap pli ca tions, pro grammed with a high level lan guageto drivethel/0 ports directly. The equip ment sat isfies su per vi sion and con trol requirements in aquacultureponds through theautomatic wa ter level mea surements.
\end{abstract}

Keywords: Level mea surement, aquacultureponds, ul tra sonic, evap o ra tion.

\section{Introducción}

Actualmente, existe un notable incremento en la actividad acuícola, la tendencia incluso es superar la pesca en la producción de alimentos
(Anón, 2004), por ello, hay necesidad de tener mayor supervisión en los estanques de cultivo de especies marinas, lo cual permita mejorar las condiciones ambientales de los cultivos. Una parte fundamental para la supervisión y control de los 
Medidor ultrasónico de nivel de agua para estanques

estanques, es medir los cambios en el nivel del agua, producidos por la evaporación y filtraciones que pueden crear concentraciones indeseables; por ejemplo, de salinidad y amoniaco.

El Centro de Investigaciones Biológicas del Noroeste (CIBNOR), realiza investigación y desarrollo de tecnología para cultivar diversas especies marinas en estanques supralitorales y tanques (Figura 1). El sistema de medición de nivel de agua, descrito en el presente trabajo, pretende contribuir a la automatización en la operación de los estanques, y así, aumentar la rentabilidad en la producción.

Si bien existen en el mercado diversos medidores de nivel, normalmente son costosos y no necesariamente diseñados para esta aplicación. De acuerdo a su principio de operación, básicamente los medidores pueden ser de presión, mecánicos de polea y ultrasónicos (Anón, 2005a, b y c).

El uso de tecnología reciente en el campo de la electrónica con componentes integrados a gran escala y que operan de manera independiente; como por ejemplo, un termómetro digital encapsulado en un montaje T0 92, cuya medición de la temperatura se hace a través de una sola terminal, permite desarrollar equipos modulares y altamente sofisticados de manera económica, precisa y confiable, tal como se muestra en esta aplicación.
Al margen de satisfacer la necesidad de este sistema de medición en tiempo real, el presente trabajo muestra la aplicación y versatilidad de varios componentes electrónicos que utilizan diversos protocolos de comunicación, los cuales permiten integrar diferentes capacidades de medición, supervisión y control de multiples variables y parámetros de interés. Una descripción sintética de las características principales del STAMP, así como de los protocolos 12C, 1-Wire y Bluetooth se presenta en el Apéndice 1.

\section{Desarrollo}

El sistema propuesto (Figura 2) está compuesto de la unidad remota instalada en el sitio de interés y la unidad local dentro de un laboratorio cercano, ambas desarrolladas con el microcontrolador STAM P BS2p (Edwards, 2001).

\section{Unidad remota}

Contenida en un módulo para intemperie que consta de un microcontrolador, un transductor ultrasónico piezoeléctrico de $40 \mathrm{kHz}$, un sensor de temperatura, un acondicionador de señal, una pantalla de cristal liquido y un transreceptor inalámbrico de $2.4 \mathrm{Ghz}$.

El microcontrolador genera un tren de pulsos de $40 \mathrm{kHz}$ con una duración programable entre 100 y $300 \mu$ s (Figura 3), la cual es amplificada por el

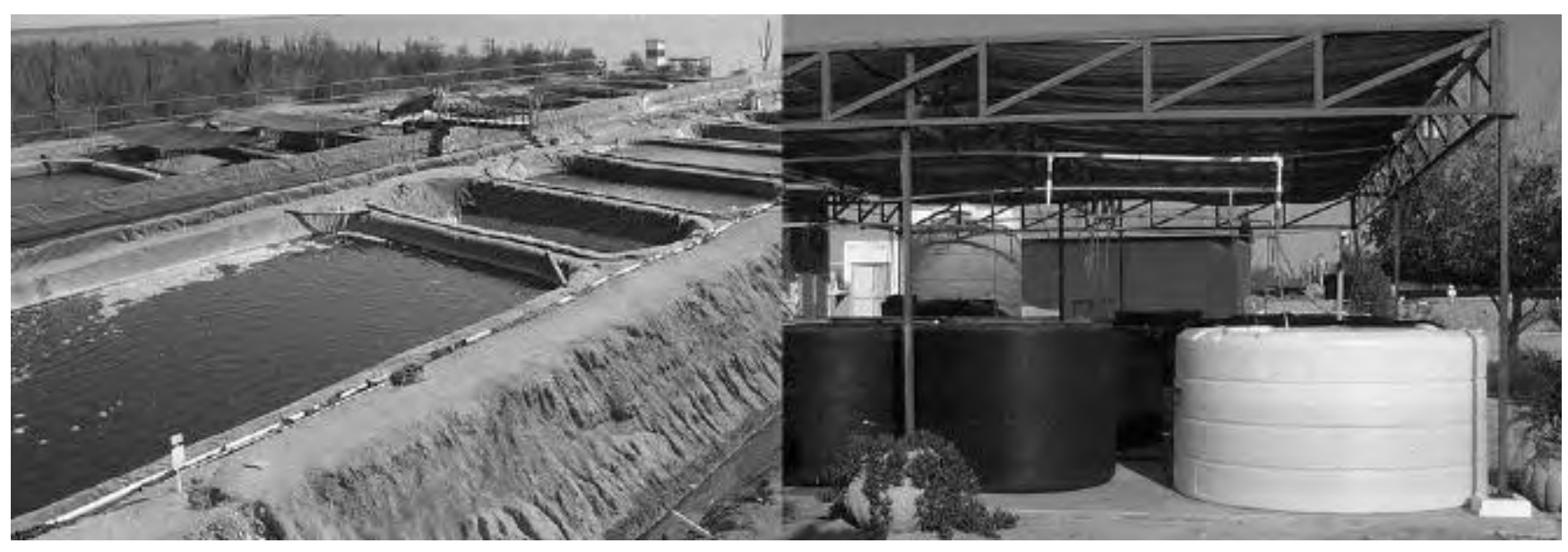

Figura 1. Estanques supralitorales y tanques de cultivo acuícola 
acondicionador de señal que consta de un amplificador operacional no inversor conectado a un transformador de acoplamiento para obtener un pulso de $160 \mathrm{Vpp}$ en el transductor (Figura 4), el cual produce una onda de presión ultrasónica entre 0.0346 y $0.1039 \mathrm{~m}$, a una temperatura ambiente de $300 \mathrm{~K}$ y de acuerdo a la duración del tren de pulsos programada.

La velocidad del sonido varía con la temperatura (Benson, 2004) de acuerdo a la ecuación:

$$
\mathrm{v}=\sqrt{\mathrm{gRT}}
$$

Donde ves la velocidad del sonido en el aire $(\mathrm{m} / \mathrm{s})$, yes la relación de calor específico (1.4 para el aire a nivel del mar), $R$ es la constante universal de los gases $\left(286 \mathrm{~m}^{2} / \mathrm{s}^{2} / \mathrm{K}\right.$ para el aire) y $\mathrm{T}$ es la temperatura $(\mathrm{K})$.

La onda de presión del pulso de trasmisión al chocar con la superficie reflejante produce un eco, que es recibido por el transductor y captada por el receptor, que también recibe el pulso de trasmisión. El voltaje del eco recibido en el transductor, depende de la distancia y de la densidad del blanco, así como de la potencia de transmisión y la sensibilidad de recepción (Figura 5). Este voltaje es amplificado a saturación por un

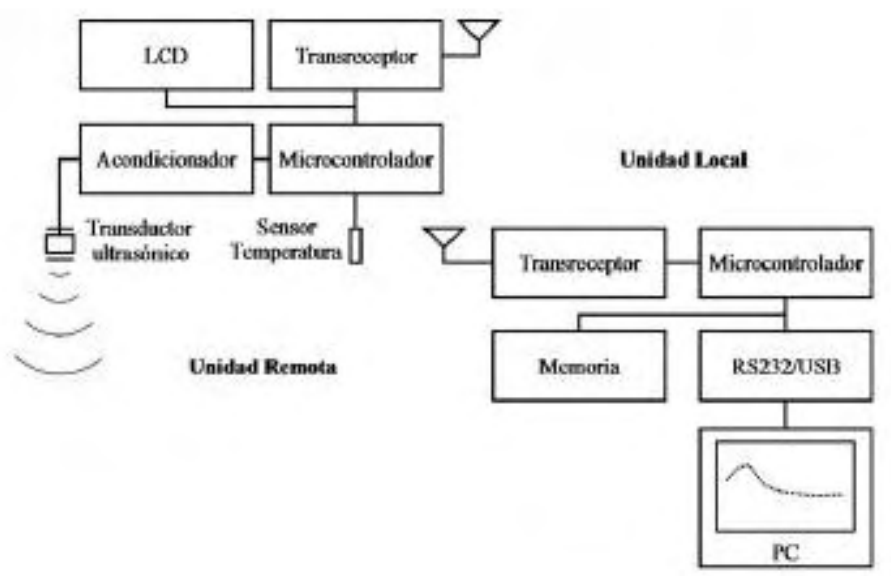

Figura 2. Componentes del medidor ultrasónico de nivel de agua para estanques

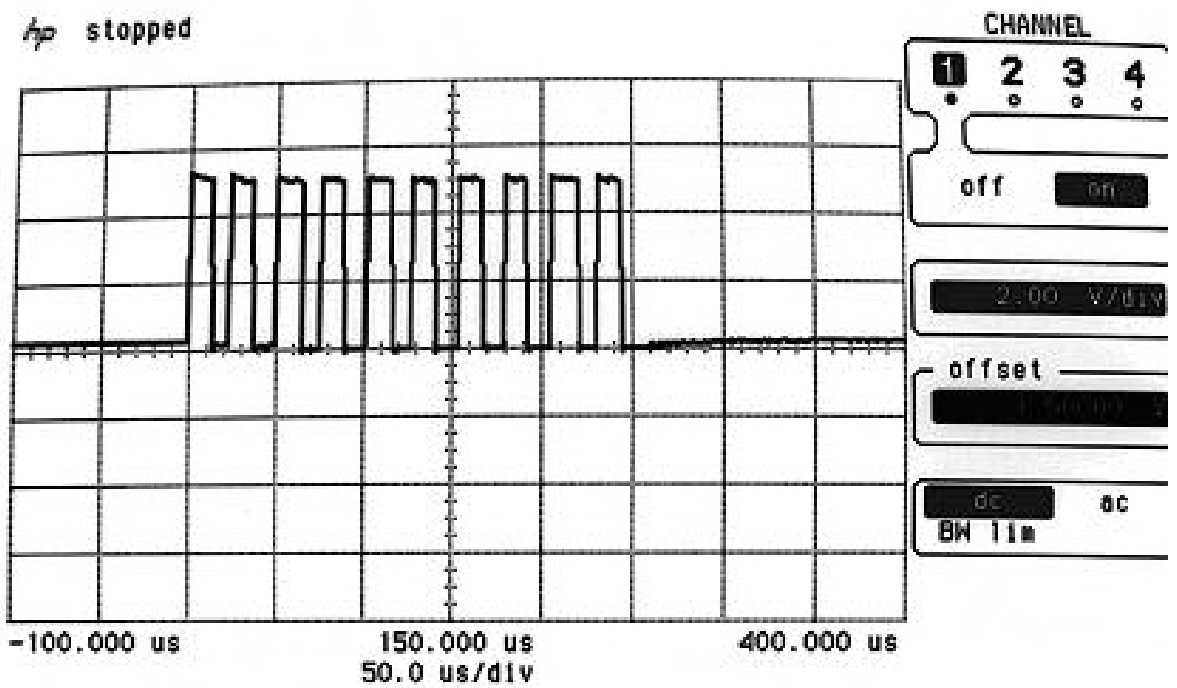

Figura 3. Tren de pulsos de trasmisión producidos por STAM P BS2p 
amplificador operacional (Figura 6), cuya salida se recorta a $5 \mathrm{~V}$ y se conecta a dos multivibradores monoestables, el primero es disparado por el pulso de inicio de trasmisión, el cual sirve para mantener su estado inestable por un tiempo constante, lo que permite eliminar el tren de la trasmisión. Después, al caer a su estado estable se produce un flanco que dispara al segundo multivibrador a su estado inestable, el que a su vez, es llevado a su estado estable (reset) por el eco (Figura 7), de esta manera, la suma de los dos tiempos de salida de los monoestables determinan el doble del tiempo de vuelo time of flight o TOF), entre el transductor y el blanco, en el caso mostrado, el blanco se encuentra a $5.8 \mathrm{~ms}$ del transductor ultrasónico.

El microcontrolador mide el ancho del pulso de segundo monoestable con una resolución de 0.8 $\mu \mathrm{s}$, la ecuación 2, permite calcular la distancia entre el transductor ultrasónico y el blanco como función del tiempo:

$$
d=v \frac{t}{2}
$$

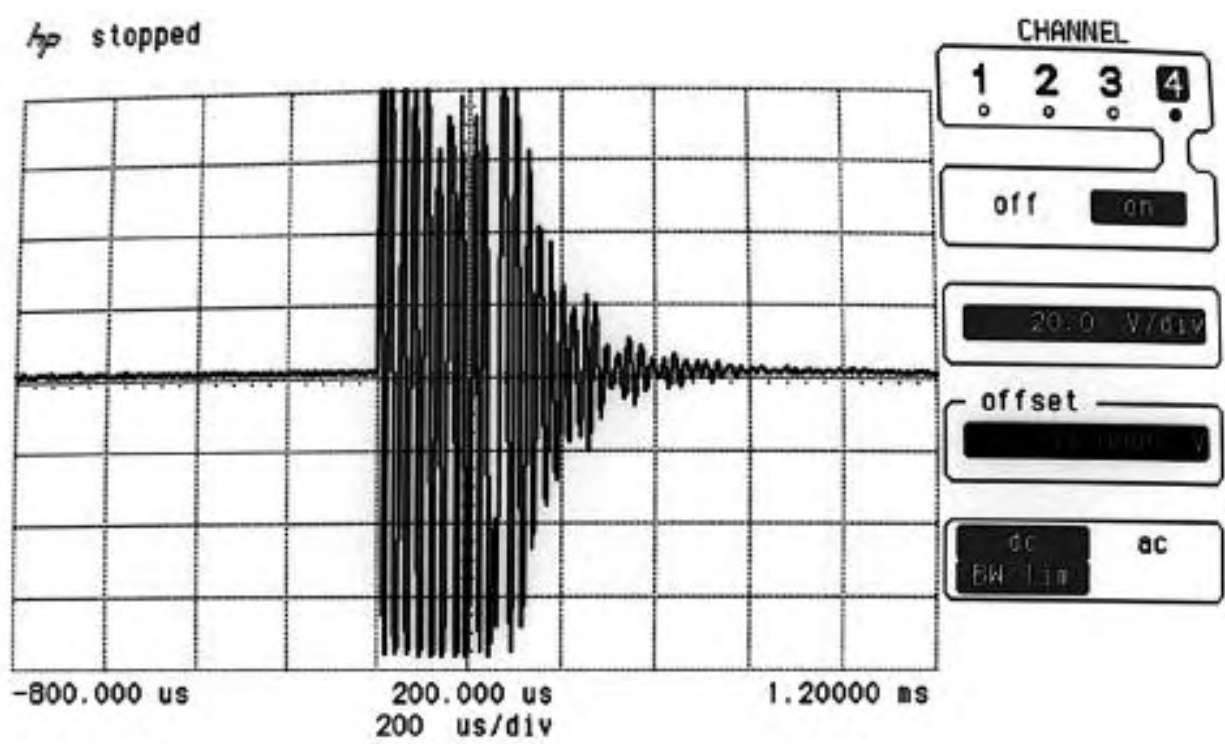

Figura 4. Pulso de trasmisión en la salida del transductor

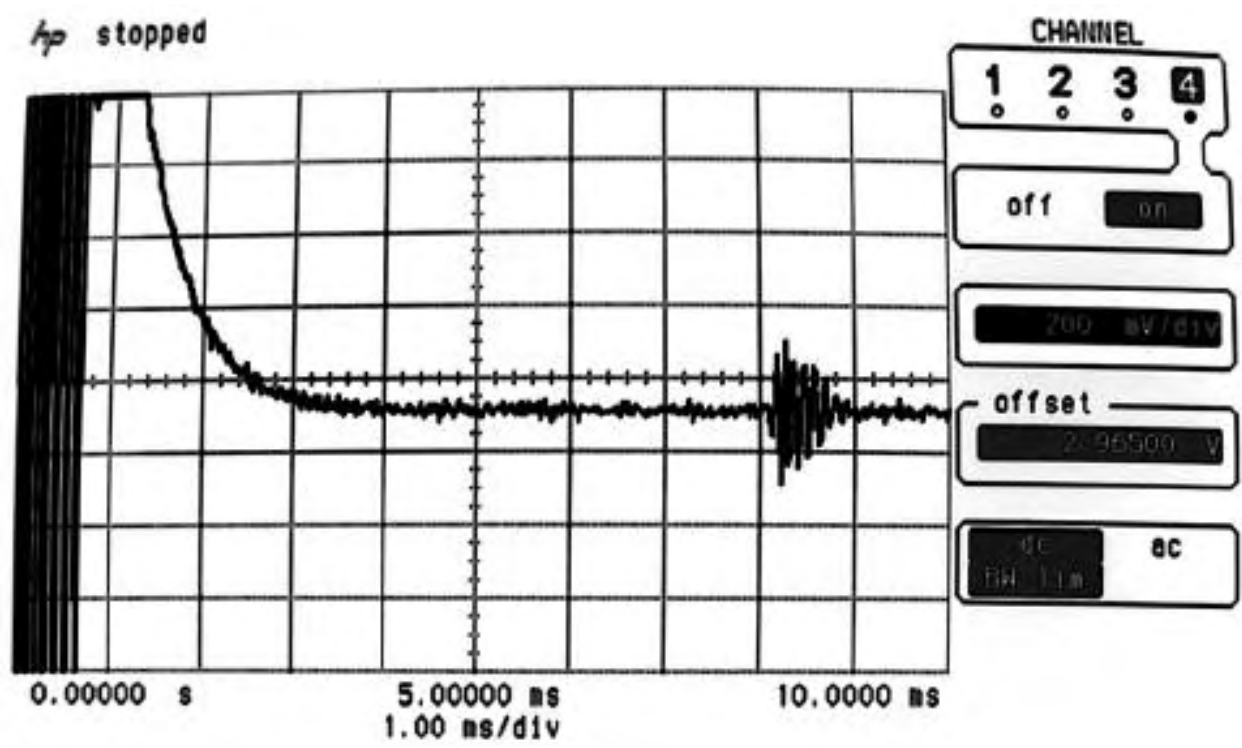

Figura 5. Pulso de trasmisión en el receptor y eco 


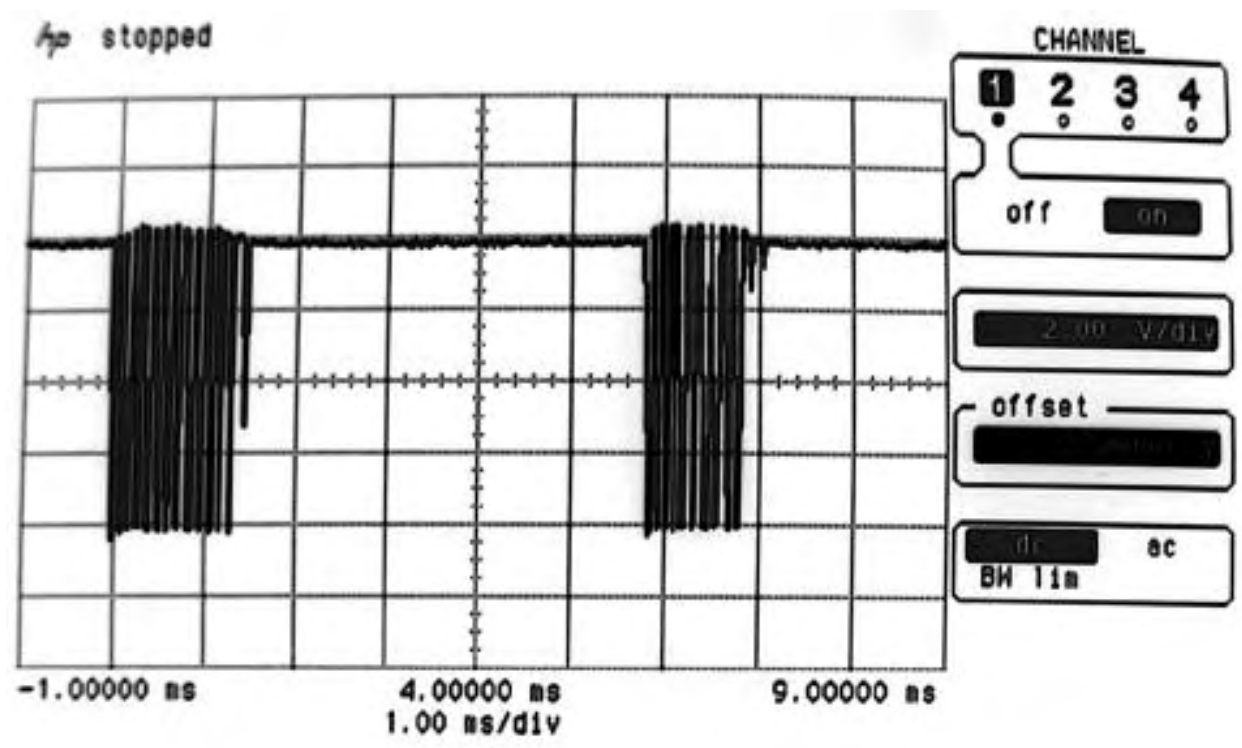

Figura 6. Saturación del pulso de trasmisión y el eco

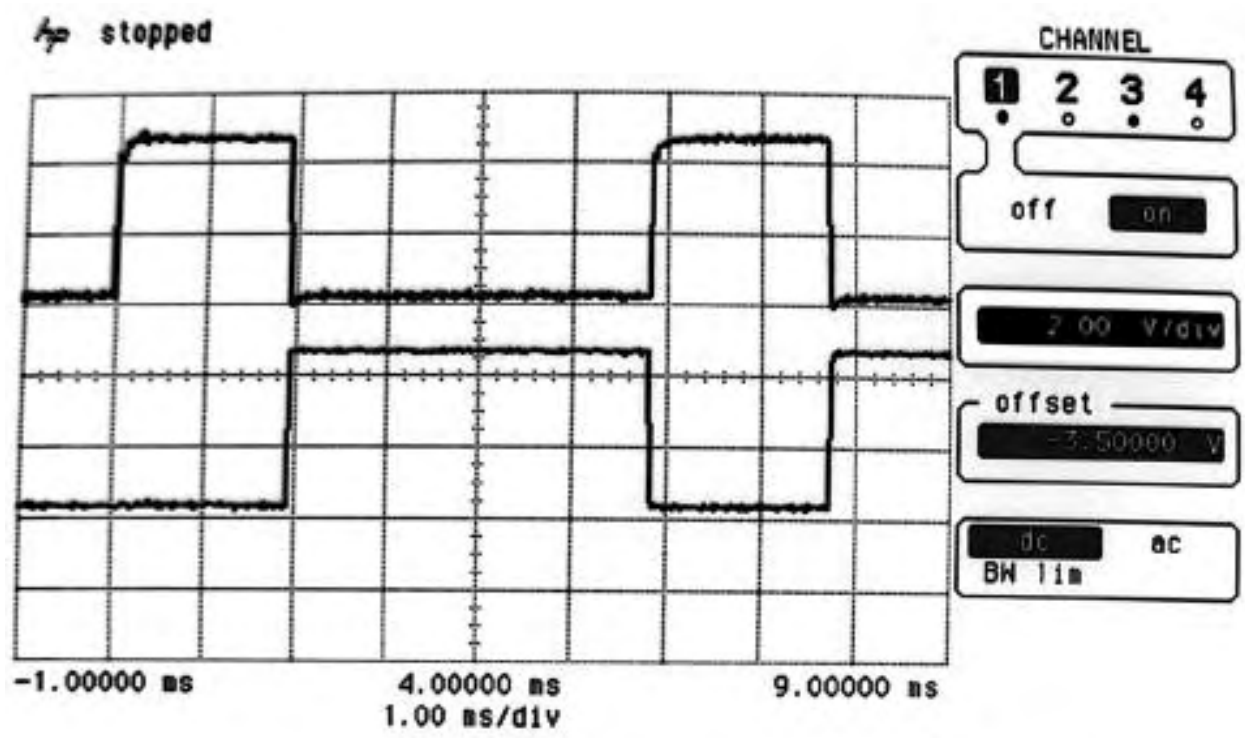

Figura 7. Señal de salida de los multivibradores monoestables para medir el tiempo de vuelo

Donde $d$ es la distancia $(m)$ recorrida por el sonido y tes el tiempo (s) total que se obtiene por la suma de los tiempos de ambos multivibradores.

Dado que la velocidad del sonido está en función de la temperatura del medio de propagación, es necesario medirla para compensar su variación de acuerdo a la ecuación 1.

Utilizando las ecuaciones 1 y 2 se puede calcular que a una temperatura ambiente de $300 \mathrm{~K}$ y una resolución en la medición de la temperatura de \pm 0.1 $K$, combinado con la resolución de tiempo de $\pm 0.8 \mu \mathrm{s}$ del microprocesador, permiten una resolución en la medición de la distancia al blanco del orden de \pm $0.0018 \mathrm{~m}$ para la distancia máxima de diseño del sistema, que es de $10 \mathrm{~m}$. Por lo que un sensor de temperatura con una resolución mayor de $\pm 0.1 \mathrm{~K}$, satisface el requerimiento teórico electrónico en la resolución de la distancia. El termómetro digital seleccionado es el DS1822 que tiene una resolución de $2^{-4} \mathrm{~K}$ en 12 bits, este dispositivo se comunica con el microcontrolador mediante el protocolo serial 1-Wire ${ }^{\circledR}$ (Stevens, 1999). La medición de temperatura y tiempo de vuelo se lleva a cabo de manera consecutiva e instantánea. 
Medidor ultrasónico de nivel de agua para estanques

La unidad remota también cuenta con una pantalla de cristal liquido HD-44780 de $2 \times 16$ caracteres para la supervisión del correcto funcionamiento del medidor de nivel, ya que muestra la información en tiempo real. La operación de esta pantalla se efectúa mediante tres comandos implementados en el microcontrolador BS2p, que permiten la comunicación directa a través de tres líneas de control y cuatro de datos.

Un dispositivo para la transmisión-recepción de información mediante el protocolo estándar Bluetooth ${ }^{\circledR}$ (Anón, 2005d), permite establecer un enlace inalámbrico para monitorear en tiempo real la variación del nivel en el estanque, producida por la evaporación y filtraciones. EI alcance del enlace electromagnético inalámbrico puede ser de hasta $1.2 \mathrm{~km}$ en línea de vista, con antena externa tipo bipolar o datch, y de $100 \mathrm{~m}$ con la antena interna integrada (clase 1). La pantalla de cristal líquido y el trasmisor, se colocaron fuera del módulo para intemperie (Figura 8), para facilitar la lectura y supervisión de la correcta operación del equipo.

Para medir la evaporación o el nivel del agua en los estanques acuícolas, el microcontrolador está programado para recibir un comando de toma de muestra que incluye el tiempo de vuelo y la temperatura ambiente. Esta información es transmitida hacia la unidad local.
La alimentación de esta unidad se proporciona mediante una batería recargable de $12 \mathrm{Vdc}$ con una capacidad de $5 \mathrm{Ah}$. Se regula el voltaje a $9 \mathrm{Vdc}$ para la parte ultrasónica e inalámbrica y a $5 \mathrm{Vdc}$ para el resto de los componentes electrónicos. EI consumo de corriente es de $70 \mathrm{~mA}$ cuando está encendido, de $100 \mathrm{~mA}$ cuando se establece la comunicación remota con la unidad local y cuando transmite el pulso de ultrasonido de 105 mA.

\section{Unidad local}

Consta de un microcontrolador, una memoria de estado sólido EEPROM y un transreceptor inalámbrico de $2.4 \mathrm{GHz}$, así como de una conexión USB para comunicarse con una computadora personal (PC).

En la computadora personal se encuentra instalado el programa ejecutable desarrollado en el lenguaje de programación Matlab 7.0. En el cual se establece la frecuencia de medición del tiempo de vuelo y de temperatura ambiente, así como la sincronía para establecer la comunicación entre el puerto USB, el microcontrolador y el transmisor inalámbrico. Este programa se activa mediante una interfaz gráfica del usuario (GUI) que permite a través del teclado o del ratón la configuración del puerto e inicio de la conexión, la selección del intervalo de muestreo y la presentación gráfica y numérica de la información recibida en tiempo real (Figura 9).

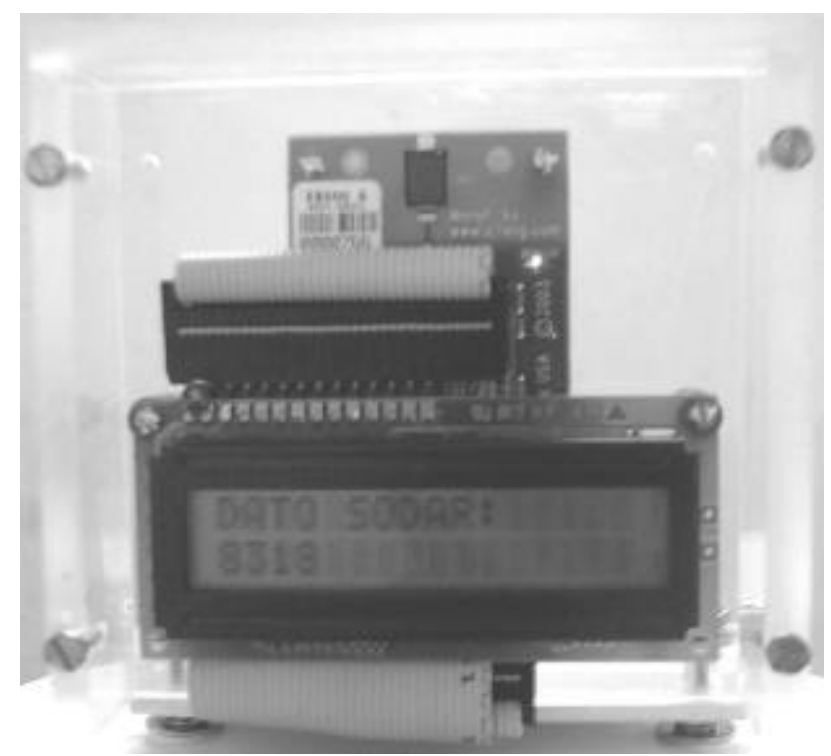

Figura 8. Detalle del trasmisor y de la pantalla de cristal liquido de la unidad remota 


\section{J. Gutiérrez y M.A. Porta-Gándara}

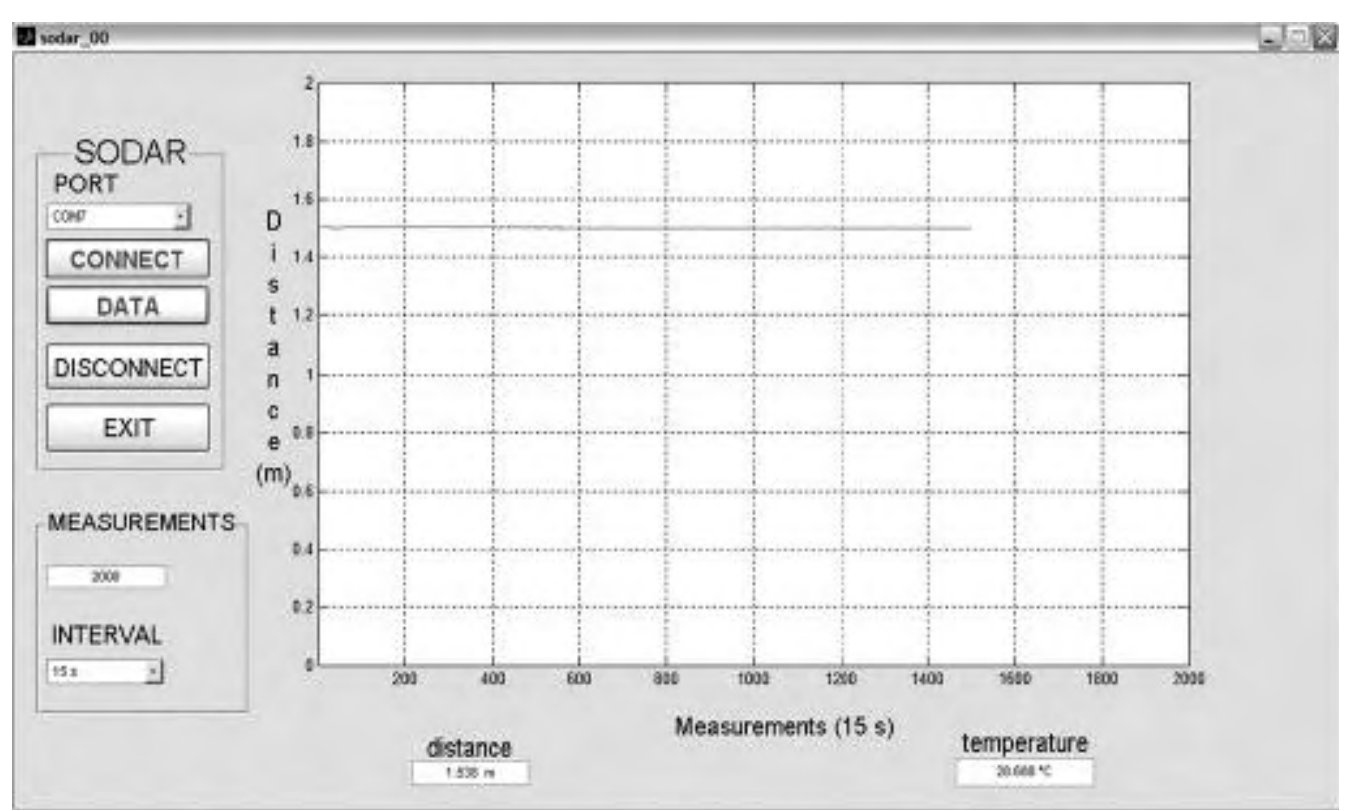

Figura 9. Pantalla de M atlab para el monitoreo en tiempo real

El cálculo de la distancia se hace mediante el algo ritmo programado con las ecuaciones (1) y (2) respectivamente, en este caso, el instrumento está diseñado para registrar cambios de nivel del agua de hasta $10 \mathrm{~m}$ con una resolución de $\pm 0.003 \mathrm{~m}$. La distancia calculada se almacena en un archivo de hojas de cálculo con extensión xls para su uso posterior, en donde se incluyen los datos recibidos, la fecha y hora.

El microcontrolador recibe el inicio de comunicación del puerto USB y se establece el enlace con la unidad remota para transmitir el comando de toma de muestra, y queda en espera de recibir los datos correspondientes. Una vez que se reciben los datos se transfieren al programa en tiempo real y se almacenan en una memoria EEPROM de 32 kB comunicada mediante el protocolo serial I2C (Stevens, 1999). Esta memoria permite el respaldo independiente de información de la computadora de hasta 16,384 valores, ya que cada dato ocupa dos bytes.

La alimentación de esta unidad se proporciona mediante una fuente de $12 \mathrm{Vdc}$ con una capacidad de $500 \mathrm{mAh}$. Se regula a $9 \mathrm{Vdc}$ para la parte inalámbrica y $5 \mathrm{Vdc}$ para el microcontrolador y la memoria EEPROM. El consumo de corriente es de $60 \mathrm{~mA}$ cuando está encendido y de $90 \mathrm{~mA}$ cuando se establece la comunicación local con la unidad remota.

\section{Resultados}

El sistema se ha probado de manera intensiva, tanto en laboratorio como en campo, para evaluar su desempeño bajo diferentes condiciones ambientales.

\section{Pruebas en laboratorio}

Se llevaron a cabo diferentes pruebas en laboratorio (Figura 10), para establecer la precisión del instrumento a diferentes temperaturas y medir de manera real su resolución, utilizando una distancia constante y conocida entre el transductor y el blanco.

Los resultados (Figura 11) muestran la variación de la medición de la distancia con respecto a los cambios de la temperatura ambiente de 29.8 a $33^{\circ} \mathrm{C}$, aproximadamente. Como se puede observar, el cambio en la distancia medida compensada en temperatura es de $0.0012 \mathrm{~m}$, en un intervalo de 2000 lecturas a una frecuencia de 15 s. La distancia fija al blanco, se midió por un extensómetro de tres metros de longitud y una resolución de un milímetro, siendo ésta de $1.5 \mathrm{~m}$. Para esta prueba resulta relevante la distancia fija entre el transductor y el blanco y no la exactitud, debido a que se desea medir la precisión y resolución del instrumento. 


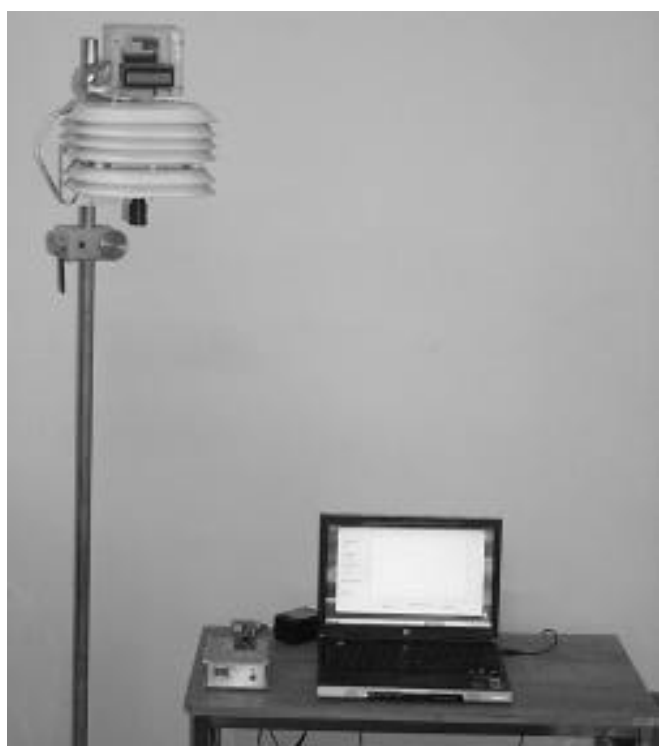

Figura 10. Unidad remota y local del medidor de nivel ultrasónico en laboratorio
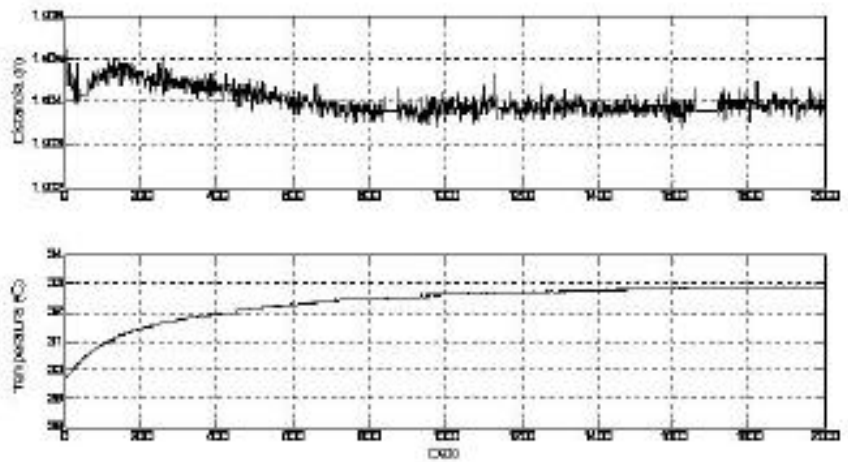

Figura 11. Resultados de la medición de una distancia fija y temperatura vari able en laboratorio

Como puede observarse entre el dato 0 y el dato 400 , existe la mayor pendiente positiva en el cambio de la temperatura ambiente y entonces el sensor de temperatura no alcanza el valor final asintótico de $95 \%$. Esto genera una fluctuación de entre 1.504 y $1.505 \mathrm{~m}$ en el valor de la distancia medida. A partir del dato 400, la pendiente de la temperatura ambiente disminuye, por lo tanto, la fluctuación en la medición de la distancia es menor.

\section{Pruebas de campo}

La medición exper i mental se desarrolló en la zona de tanques de cultivo (Figura 12). Para poder evaluar el desempeño del equipo en campo, se seleccionó un tanque de cultivo de microalgas de $5000 \mathrm{~L}$ de capacidad y un tirante aproximado de un metro, medido con el extensómetro antes mencionado. Se eligió este contenedor, ya que no existen fugas de agua debidas a filtraciones y solamente el cambio de nivel es producto de la evaporación.

Para el cultivo de las microalgas, primeramente se llenó el tanque y se dejó reposar el agua por un perío do de $24 \mathrm{~h}$, en el cual se efectuó una primera fase del experimento de campo. El resultado de la evaporación (Figura 13) muestra un cambio en el nivel del tirante de agua aproximadamente de 0.99 


\section{J. Gutiérrez y M.A. Porta-Gándara}

a $0.975 \mathrm{~m}$, que corresponde alrededor de $15 \mathrm{~L} / \mathrm{m}^{2}$. En la gráfica de distancia contra tiempo se aprecian fluctuaciones en el nivel del agua de hasta $0.004 \mathrm{~m}$ producidas por el viento, cuya intensidad fue de casi $3 \mathrm{~m} / \mathrm{s}$ al inicio de la prueba.

En la segunda fase del experimento, las microalgas son sembradas al tanque de cultivo y se conecta un aereador para proporcionar el oxígeno requerido para su crecimiento. El resultado de la evaporación (Figura 14) se ve influenciado por el cambio de nivel producido por el burbujeo del aereador y el viento; como puede observarse en la gráfica, las fluctuaciones del nivel de agua son de hasta $0.025 \mathrm{~m}$. El cambio resultante en el nivel del agua en la prueba a lo largo de $24 \mathrm{~h}$, fue de una diferencia de $0.018 \mathrm{~m}$ que corresponde alrededor de $18 \mathrm{~L} / \mathrm{m}^{2}$. Puede apreciarse que el burbujeo produce un incremento en la evaporación, al comparar los resultados de ambas fases.

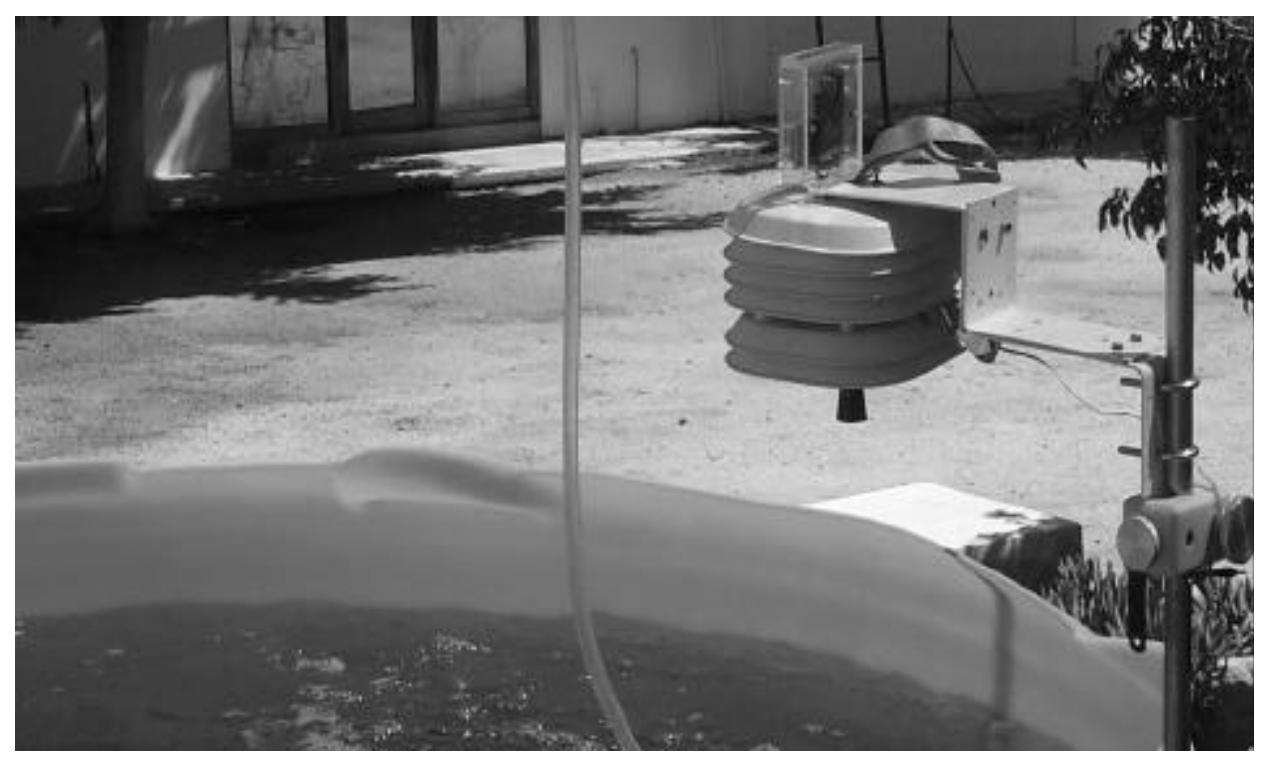

Figura 12. Unidad remota en operación en un tanque acuícola
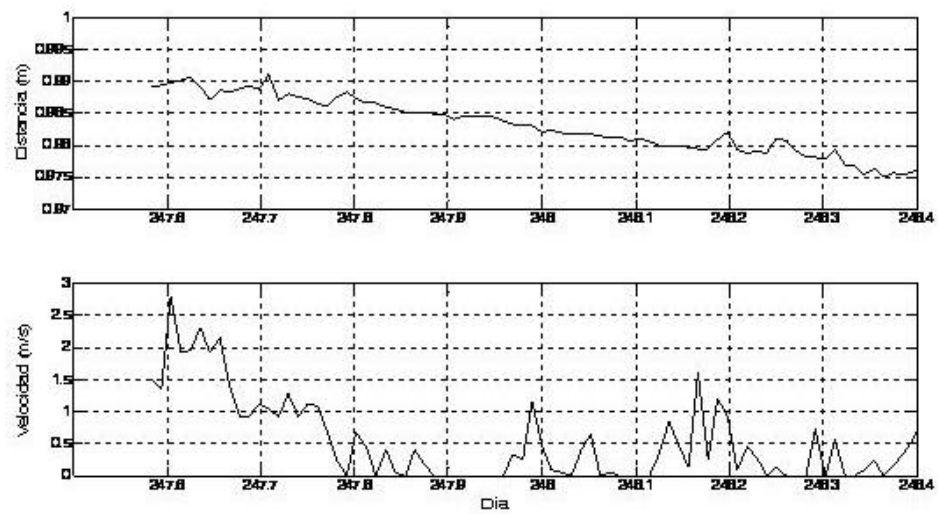

Figura 13. Resultados de la medición de nivel en el tanque acuícola sin burbujeo y velocidad de viento 

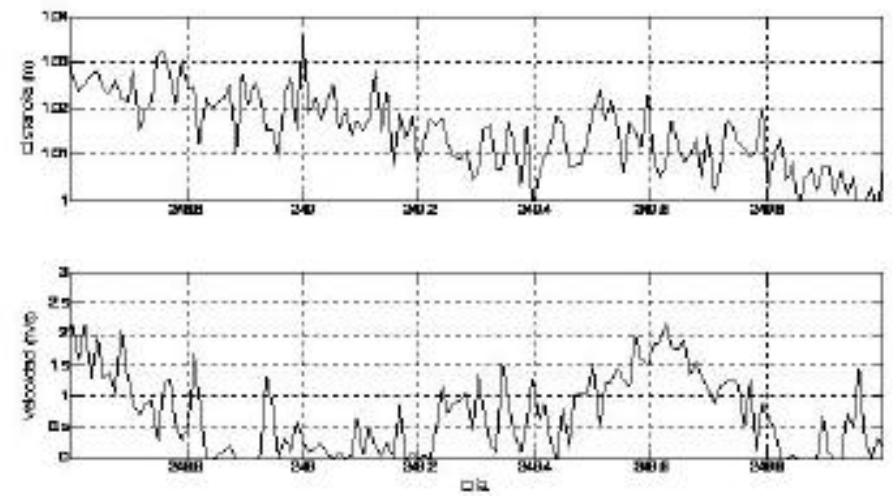

Figura 14. Resultados de la medición de nivel en el tanque acuícola con burbujeo y velocidad de viento

\section{Conclusiones}

El sistema implementado es autónomo y portátil, de fácil instalación en una diversidad de escenarios posibles. Por su característica de equipo telemétrico no requiere de instalaciones físicas fijas. Es práctico operar el sistema en campo, tanto la unidad local como la remota, mediante una computadora portátil personal, o bien, con la unidad local dentro de una oficina o laboratorio a distancia.

El equipo se sometió a diversas pruebas tanto en campo como en laboratorio con resultados satisfactorios y confiables, por lo que el sistema cumple las expectativas de diseño y operación. La información resultante es un archivo estándar de fácil interoperabilidad con programas computacionales de uso común para el procesamiento de datos.

Una de las características de este equipo es el haber logrado el aumento en la resolución con un medidor ultrasónico, que normalmente es $\pm 0.01 \mathrm{~m}$, únicamente equipos de más alta tecnología como los que usan láser tienen resoluciones parecidas 0 superiores a este equipo, pero son de mayor costo.
El uso de un microcontrolador como el STAM P BS $2 p$, permite tener una plataforma versátil, simple y práctica para el desarrollo de diferentes aplicaciones en el campo de la instrumentación y control. A diferencia del uso de microcontroladores de más bajo nivel como pueden ser los PICs o A tmel, el STA M P integra una serie de instrucciones que permiten la comunicación directa por medio de los diferentes protocolos integrados en su lenguaje de programación.

En este trabajo se ha mostrado una parte del potencial de este tipo de microcontrolador para desarrollar sistemas integrados robustos y de muy bajo costo, a partir de equipos originales manufacturados (i.e., OEM), tal como microcontroladores, computadoras compactas, dispositivos de comunicación y sensores diversos. Con esta arquitectura es factible añadir sensores específicos para diferentes tareas de medición, supervisión y control. Mediante los acondicionadores de señal adecuados, se logra el crecimiento modular a sistemas más complejos y completos, sin tener que rediseñar to do el sistema.

Una mejora del sistema puede hacerse mediante un sensor de temperatura que tenga un tiempo de respuesta menor, como podría ser un circuito termopar digital (v.g. MAX667), que permitiría mejorar la compensación de temperatura 
de la velocidad del sonido, y así poder aumentar la resolución en la medición de la distancia mediante ultrasonido.

\section{Apéndice 1}

\section{Microcontrolador STAM P BS2p}

Este microcontrolador manufacturado por PARALLAX, Inc, está basado en la familia SX de controladores de comunicaciones configurables, fabricados con tecnología CMOS avanzada, combinados con arquitectura RISC que permite altas velocidades de cómputo con control flexible y eficiente en la manipulación de datos, a través de sus puertos de entrada/salida. EI BS2p está encapsulado en un empaque de 24 terminales (DIP 24-pins), es alimentado entre 6-9 Vdc. Un regulador de voltaje proporciona los $5 \mathrm{~V}$ para alimentar al $B S 2 p$, tiene una EEPROM de $2 \mathrm{~kb}$ y $32 \mathrm{~b}$ de memoria RAM , se programa en lenguaje $P B$ asic que es almacenado permanentemente en la EEPROM. El programa definido por el usuario es cargado desde una PC a través de un puerto serial o USB. EI BS2p tiene 16 puertos programables de entrada/ salida, cada terminal proporciona una corriente máxima de $30 \mathrm{~mA}$.

El PBasic tiene un conjunto de 56 instrucciones que permiten hacer las funciones más elementales de un lenguaje BASIC estándar y operar directamente los siguientes protocolos: Serial RS232, 1-Wire ${ }^{\circledR}$ y $12 C \circledR$, así como la implementación de comandos para la operación directa de pantallas de cristal líquido.

\section{Protocolo I2C}

El medio de control que proporciona un enlace de comunicación entre circuitos integrados en un sistema, se conoce como I2C (Inter-IC bus), desarrollado por Philips Semiconductors a principios de 1980. Mediante un protocolo de comandos de comunicación de dos cables, se ha creado un estándar mundial para los sistemas de control. Este protocolo se aplica a una diversidad de circuitos, como convertidores $A / D, C O D E C s$, microprocesadores, sensores, entre otros. Las velocidades de trasmisión alcanzan 3.4 Mbps, Dos líneas conectan a todos los circuitos integrados en el sistema y cualquier dispositivo $12 \mathrm{C}$ puede ser conectado e intercambiar información entre el dispositivo maestro y esclavo. El esquema de direccionamiento, elimina la necesidad de circuitos decodificadores.

Cada transferencia $12 \mathrm{C}$ se efectúa con una condición de inicio (Start Condition) y termina con una condición de alto (Stop Condition). Entre estas dos condiciones se transfiere un byte con la identificación única del dispositivo esclavo, seguido por un byte de direccionamiento y otro de dato. Inmediatamente después de que cada byte es transmitido, durante un ciclo de reloj una señal de reconocimiento (Acknowledge).

Los diseñadores pueden pasar rápidamente del diseño de escritorio al circuito final. ElI2C también ahorra espacio y costos, haciendo el circuito impreso de menor tamaño. Actualmente, existen más de 400 dispositivos $12 \mathrm{C}$ y se añaden e incrementan anualmente entre 40 y 50.

\section{Protocolo 1-Wire}

La tecnología 1-Wire desarrollada por Dallas Semiconductors, consiste de un cable que sirve para alimentar y comunicar de manera serial asíncrona y bidireccional a una gran diversidad de dispositivos electrónicos. Por lo tanto, sólo se requiere de una terminal de entrada/salida compartida entre múltiples dispositivos 1-Wire, los cuales tienen una identificación digital de direccionamiento única que consiste en un número de 64 bits grabado permanentemente en una ROM, donde el primer byte corresponde a un código de la familia del tipo de dispositivo y los restantes 6 bytes proporcionan 48 bits de un número único para cada dispositivo (le. $2^{48}$ circuitos posibles). Existe una gran cantidad de circuitos para medir humedad, presión barométrica, dirección de viento, radiación solar y temperaturas, entre otros.

El protocolo utiliza niveles lógicos TTL/CMOS convencionales con rangos de voltaje de 2.8 a $6 \mathrm{~V}$ $\mathrm{dc}$, tanto el maestro como los esclavos se configuran como transrecpetores al permitir un dato secuencial de bits en cualquier dirección, pero solo en una dirección al mismo tiempo. La 
Medidor ultrasónico de nivel de agua para estanques

información se transfiere en cuatro etapas de tiempo: inicialización, identificación del número único, comando y transferencia de dato.

\section{Protocolo B luetooth}

Desarrollado por ERICSSON, Bluetooth es un estándar industrial inalámbrico para comunicar redes personales confinadas, permite intercambiar información entre diferentes dispositivos como asistentes digitales personales PDAs), teléfonos móviles, computadora portátil, impresoras, cámaras digitales, equipos de sonido, entre otros. En la actualidad, existen tres clases disponibles de potencia: clase 3 de $1 \mathrm{~mW}$ que permite una comunicación hasta $1 \mathrm{~m}$, clase 2 de $2.5 \mathrm{~mW}$ con un alcance de $10 \mathrm{~m}$ y clase 1 de $100 \mathrm{~mW}$ hasta $100 \mathrm{~m}$; sin embargo, existen antenas externas que permiten la comunicación hasta $1200 \mathrm{~m}$.

El protocolo opera en una banda libre ISM (Industrial Scientific and $\mathrm{M}$ edical) de radiofrecuencia a $5.8 \mathrm{GHz}, 2.4 \mathrm{GHz}$ y $900 \mathrm{MHz}$. Para prevenir interferencia con otros protocolos que utilizan la banda de $2.45 \mathrm{GHz}$, el Bluetooth divide la banda en 79 canales de $1 \mathrm{MHz}$ de ancho de banda.

Un dispositivo Bluetooth tiene el rol de maestro y puede comunicarse con más de siete dispositivos con el rol de esclavos. En cualquier tiempo, los datos pueden ser transferidos entre el maestro y un esclavo; pero el maestro cambia rápidamente su comunicación con los demás esclavos, este esquema de comunicación se conoce como piconets. Cada dispositivo tiene un número de identificación de 12 caracteres para establecer la comunicación; sin embargo, éste puede estar configurado a responder a cualquier petición de conexión.

El rey danés y noruego Harald Blåtand (Harold Bluetooth en inglés), alrededor del año 1000 DC, unificó las tribus guerreras de Dinamarca, Noruega y Suecia, ERICSSON toma este nombre por ser el Bluetooth in protocolo unificador de diferentes tecnologías.

\section{Referencias}

Anón (2004). The State of World Fisheries and Aquaculture (SOFIA). The Food and Agriculture Organization, Fisheries Departament. Rome, Italy, Part 1, pp. 65.

Anón. (2005a). SIAP Bologna: Water Level Recorder 5794.http://www.siap.com

Anón. (2005b). Applied Microsystems Inc.: Water Level Recorder PA-10. http://www.appliedmicrosystems.com

Anón. (2005c). Aanderaa Instruments: Water Level Recorders WLR7. http://www.aanderaa.no

Anón. (2005d). The Official Bluetooth Membership Site. http://www.bluetooth.org

Edwards S. (2001). Programming and Custom izing the Basic Stamp Computer. McGraw-Hill, New York, NY, pp. 331.

Benson T. (2004). Glenn Learning Tech nol ogies Homepage. Glenn Research Center, NASA. http://www.grc.nasa.gov/WWW/K-12/airplan e/sound.html

Stevens R. (1999). Serial PIC'n: PIC Microcontroller Serial Communications. Square 1 Elec tronics, Hayden, Idaho, pp. 526.

\section{Semblanza de los autores}

Joaquín Gutiérrez. Es ingeniero en comunicaciones y electrónica por el Instituto Politécnico Nacional, obtuvo el grado de doctor en inteligencia artificial por el Instituto Tecnológico y de Estudios Superiores de Monterrey. Actualmente se desempeña como investigador en el Centro de Investigaciones Biológicas del Noroeste, S.C.

M iguel Ángel Porta-Gándara. Es ingeniero en comunicaciones y electrónica por el Instituto Politécnico Nacional, obtuvo el gra do de doctor en ingeniería por la Universidad Nacional Autónoma de México. Actualmente se desempeña como investigador en el Centro de Investigaciones Biológicas del Noroeste, S.C. 